\title{
INTERSTELLAR MOLECULE FORMATION IN GRAIN MANTLES: THE LABORATORY ANALOG EXPERIMENTS, RESULTS AND IMPLICATIONS*
}

\author{
W. HAGEN, L. J. ALLAMANDOLA and J. M. GREENBERG \\ Laboratory Astrophysics Group, Huygens Laboratory, Leiden, Netherlands
}

\begin{abstract}
Laboratory and theoretical studies have been made of the effects of ultraviolet photolysis of interstellar grain mantles which consist of combinations of hydrogen, oxygen, carbon and nitrogen - 'dirty ice'. It is shown that processes involving photolysis (photoprocessing) of interstellar grains are important during most of their lifetime even including the time they spend in dense clouds. A laboratory designed to simulate the interstellar conditions is described. This is the first time such a laboratory has been able to provide results which may be directly scaled to the astrophysical situations involving interstellar grains and their environment. The evolution of grain analogs is followed by observing the infrared absorption spectra of photolyzed samples of ices deposited at $10 \mathrm{~K}$. The creation and storage of radicals and the production of molecules occur as a result of reactions within the solid. A large number of molecules and radicals observed in the interstellar gas appear in the irradiated ices. Energy released during warm-up is seen from visible luminescence and inferred from vapor pressure enhancement which occurs during warming of photolyzed samples relative to unphotolyzed samples. The evolution of a grain and its role as a source as well as a sink of molecules is pictured as a statistical process within dense clouds. The gradual accretion on and photolysis of an individual grain provides the stored chemical energy the release of which is sporadically triggered by relatively mild events (such as low velocity grain-grain collisions) to produce the impulsive heating needed to eject or evaporate a portion of the grain mantle. An extremely complex and rather refractory substance possessing the infrared signatures of amino groups and carboxylic acid groups and having a maximum mass of 514 amu has been produced at a rate corresponding to a mass conversion rate of interstellar grains of between $2 \%$ and $20 \%$ in $10^{7} \mathrm{yr}$. The shape and position of the astronomically observed $3.1 \mu \mathrm{m}$ band is duplicated in the laboratory and is shown to be a natural consequence of the processing of grain mantles.
\end{abstract}

\section{Introduction}

At the Astrophysics Laboratory of Leiden University we have developed an experimental system which creates the relevant interstellar analog conditions leading to the formation of a wide range of molecules and their injection into interstellar space as a consequence of the evolution and photoprocessing of grain mantles. The results on ultraviolet produced changes in chemical composition, storage of radicals and vapor pressure enhancement due to the heat released by the combination of reactive species stored in the complex mixtures all lend support to the picture that the interstellar grain mantles are not static sinks capturing all molecules striking them, but rather dynamic

\footnotetext{
* Invited contribution to the Proceedings of a Workshop on Thermodynamics and Kinetics of Dust Formation in the Space Medium held at the Lunar and Planetary Institute, Houston, 6-8 September, 1978.
}

Astrophysics and Space Science 65 (1979) 215-240. 0004-640X/79/0651-0215\$03.90

Copyright (C) 1979 by D. Reidel Publishing Co., Dordrecht, Holland, and Boston, U.S.A. 
centers of activity in which gas phase molecules and radicals are modified and regenerated by reactions within the grains. We are thus able to demonstrate that the prevailing theories of molecule formation which consider only gas phase and grainsurface reactions are not complete (Watson, 1976) because they have not included the role played by the interiors of interstellar dust grains in the formation of molecules.

Although the chemical composition of the grains has been a subject of interest to astronomers ever since it was first clearly established that there existed a general distribution of obscuring material in the Milky Way, the full realization of the contribution of their chemistry to the chemical composition of the interstellar medium has been slow to emerge. The suggestion by Lindblad (1935) that the grains could condense (or more properly, accrete) directly out of the gas in the interstellar medium led to the studies by ter Haar (1943) and van de Hulst (1943) on the mechanisms of grain formation. Using the then current ideas of the interstellar medium as containing only atoms, van de Hulst (1949) assumed that the relatively abundant condensible atoms $(\mathrm{O}, \mathrm{C}, \mathrm{N})$ which collided with an already existing grain would almost certainly stick and combine with the very abundant hydrogen atoms which were present on the grain surface to form, finally, the saturated molecules water $\left(\mathrm{H}_{2} \mathrm{O}\right)$, methane $\left(\mathrm{CH}_{4}\right)$ and ammonia $\left(\mathrm{NH}_{3}\right)$. Putting these together in relative cosmic abundance $(\mathrm{O}: \mathrm{C}: \mathrm{N})$ and allowing for the additional accumulation of other less abundant atomic species led van de Hulst to the notion of homogeneous 'dirty ice' grains. If we stop and think about it, this was one of the first proposals for the interstellar formation of molecules.

It took years before the advent of new infrared techniques made it possible to attempt a direct chemical identification of the grains by searching for the $3.07 \mu \mathrm{m}$ $\mathrm{H}_{2} \mathrm{O}$ ice band in heavily obscured stars (Danielson, Woolf and Gaustad, 1965). This observation gave a negative result which provided an upper bound on the amount of $\mathrm{H}_{2} \mathrm{O}$ in grains which was a factor of at least 10 less than that expected. The interpretation of this result as indicating that the 'dirty ice' model of grains was not correct was based principally on the assumption that all of the oxygen in a grain mantle should be in the form of $\mathrm{H}_{2} \mathrm{O}$. An alternative interpretation proposed at that time by one of us (J.M.G.) was that, as a result of the photodissociation of $\mathrm{H}_{2} \mathrm{O}$ in the grains by high energy ultraviolet radiation, the 'ice' band at $3.07 \mu \mathrm{m}$ could be reduced or modified. This idea remained relatively dormant until the stimulus provided by the radio detection of the surprisingly complex molecule formaldehyde $\left(\mathrm{H}_{2} \mathrm{CO}\right)$ in the interstellar gas (Snyder et al., 1969) led to the re-examination of the consequences of photodissociating not only the $\mathrm{H}_{2} \mathrm{O}$ in the grains but also any of the other molecules. The radicals produced by photodissociation of saturated molecules could then react to form entirely new and more complex molecules in the solid in ways suggested by the production of complex (prebiogenic) organic molecules in energetically stimulated mixtures of simple gases (Miller, 1953). It is interesting to note that the idea that radicals could exist in grains had been suggested as early as 1960 (Ewing et al., 1960; Donn, 1961; Greenberg, 1963). 
Since the interstellar photochemical problem is a very complex one, the only realistic way to study it is in the laboratory. An experimental program of investigation of the effects of high energy photons on analogs of interstellar ices was initiated in 1970 at the State University of New York at Albany, with the help of University funds, and a first report was presented at the American Association for the Advancement of Science December, 1970, meeting (Carey, 1970). Using frozen mixtures of water, methane and ammonia deposited and irradiated at temperatures of 28 and $42 \mathrm{~K}$ (also at $77 \mathrm{~K}$ with ethane replacing methane), products with molecular weights as high as $M>200 \mathrm{amu}$ were produced. The quantum efficiency of the process was estimated to be of the order of $0.1 \%$ (Greenberg et al., 1972).

Although we were not able to achieve laboratory temperatures as low as the mean interstellar grain temperature of $10 \mathrm{~K}$ (Greenberg, 1971), these early experimental results were encouraging.

The purpose of this paper is to describe the experimental methods developed at Leiden and their application to produce and study the effects of irradiation of low temperature grain mantle analogs. Our initial selection of chemical systems was influenced by the following criteria: astrophysical significance, ease of sample handling and simplicity of starting mixtures. The first requirement naturally limits one to initial mixtures consisting of species made up of $\mathrm{C}, \mathrm{N}, \mathrm{O}$ and $\mathrm{H}$ atoms which are known to be most abundant in the interstellar medium, while the second leads to the use of simple molecules, not atoms. The combination of these two criteria results in the initial choice of the simple molecules $\mathrm{CO}, \mathrm{H}_{2} \mathrm{O}, \mathrm{NH}_{3}$ and $\mathrm{CH}_{4}$. Very little is known about the photochemical behaviour of such mixtures at low temperatures, although all of these molecules have been studied individually in numerous photochemical investigations (Bass and Broida, 1960; Meyer, 1971; Hallam, 1973). The spectroscopic assignments made below are based largely upon the results of those previously reported low temperature matrix isolation studies in which the complexity of the starting material has been kept to a minimum. These studies are normally carried out in dilute inert gas matrices in order to promote the preferential formation and isolation of reactive species. Since the necessary and opposite requirements for the astrophysical problem are the complete removal of inert gas and increased complexity of starting mixtures, substantially different but not unrelated results are to be expected. We present some results from a variety of experiments which give a clear indication of the major phenomena associated with interstellar grains. We aim at understanding:

(1) the photoprocessing of grains,

(2) grains as a source of molecules up to a very high order of complexity and their injection into the interstellar medium subsequent to photoprocessing,

(3) grain emission and absorption characteristics from the far infrared to the ultraviolet.

Because we are dealing with systems which have not previously been subjected to laboratory experiments we expected to find, and have indeed found, a number of new and exciting phenomena. 


\section{Interstellar Conditions and the Laboratory Analog}

For the physical and chemical processes considered here we may represent the mean interstellar grain in terms of a spherical core (radius $\sim 0.05 \mu \mathrm{m}$ of some generalized silicate and/or metallic oxide) on which has been accreted out of the interstellar gas a mantle of mean radius $\sim 0.12 \mu \mathrm{m}$ (Hong, 1976; see Greenberg, 1978 for a recent review). The mantle material must consist predominantly of chemical combinations of hydrogen and the most abundant condensible species $\mathrm{O}, \mathrm{C}$, and $\mathrm{N}$. The mean lifetime of the total grain, including the refractory cores, is probably of the order of $5 \times 10^{9} \mathrm{yr}$ (Greenberg, 1979a) as based on accepted star formation rates (Oort, 1974; Field, 1978) while the mean lifetime of a grain mantle is perhaps about one tenth of this (Salpeter, 1977; Greenberg, 1979b). However, the time a grain spends in a reasonably quiescent state (although still subject to substantial ultraviolet radiation) is likely to be comparable with the time intervals between diffuse 'cloud-cloud collisions' (Spitzer, 1969): namely, about $10^{7} \mathrm{yr}$ and no less than $\sim 10^{6} \mathrm{yr}$. The mean interstellar ultraviolet radiation flux used here for photons with $6 \mathrm{eV}<E<13.6 \mathrm{eV}(2065>\lambda>912 \AA)$ is $\sim 10^{8} \mathrm{~cm}^{-2} \mathrm{sec}^{-1}$ as deduced from Habing (1968). More recent theories of the ultraviolet energy density (for example, Witt and Johnson, 1973) do not change our predictions. We have chosen the lower limit of $6 \mathrm{eV}$ photons as representative of the minimum energy required for breaking a typical chemical bond (see for example Calvert and Pitts, 1966).

Just as gas phase molecules are photodissociated by photons of sufficient energy, so are molecules imbedded in a solid. A major difference in the solid is that some of the break-up products may more readily recombine (the cage effect) while others (say $\mathrm{H}$ ) may readily diffuse through the solid and combine with another molecule or with a previously produced radical (see Bass and Broida, 1960, particularly chapter 4; Meyer, 1971; Hallam, 1973). This means that not every 'bond-breaking' photon which is absorbed by the grain necessarily produces a net photodissociation. Indicative of the significance of grain mantle photolysis as an important interstellar process is the comparison of the life cycle time of a grain mantle with the time required for the interaction of every molecular bond in the mantle with one high energy photon. With an ultraviolet flux of $10^{8} \mathrm{~cm}^{-2} \mathrm{~s}^{-1}$ the time required for the number of photons absorbed by the grain to equal the number of molecular bonds is only $\sim 200 \mathrm{yr}$ (Greenberg, 1973). We may thus conclude that even if the photolysis efficiency per photon is taken to be as small as $10^{-3}$ (a very conservative estimate), the time, $\tau_{\mathrm{ph}}$, required to leave a net $1 \%$ of radicals (a rough upper limit, see Jackson, 1959a, b; or see chapter 10 in Bass and Broida, 1960) in the mantle is still only about 1000 yrs which is quite small compared with the mantle life-cycle time $\left(\sim 10^{6}\right.$ to $\left.10^{7} \mathrm{yr}\right)$. The fact that the penetration of the photons into the volume of the grain is not uniform (because of attenuation) only increases the photolysis time scale for the deepest molecules in the grain by about a factor of 10 (see Greenberg, 1979b for a discussion of grain penetration by ultraviolet photons). 
What happens to a grain inside a dark cloud where the interior of the cloud is shielded from some of the ultraviolet photons? Here, a relevant mantle time scale is the lifetime of the particular cloud for which we consider the free-fall time, $\tau_{\mathrm{ff}}$, to be an absolute lower limit (Zuckermann and Evans, 1974; Bash, 1979).

Let us assume an average extinction curve to prevail in the vacuum ultraviolet. Let us further define $A_{\mathrm{UV}}$, the extinction at $\lambda^{-1}=8 \mu \mathrm{m}^{-1}(\lambda=1250 \AA, E \approx 10 \mathrm{eV})$, as representative of the mean extinction for photons with $\lambda<2000 \AA(E>6 \mathrm{eV})$. In this case $A_{\mathrm{UV}} / A(V) \leqslant 3$ in a dark cloud. This is most probably an upper bound for two reasons: (1) the classical grains producing the visual extinction are expected to be larger than normal in dense clouds so that their relative contribution to the total extinction is greater, (2) there is a possible accretion of the very small grains producing the far ultraviolet extinction onto the classical grains (see Greenberg, 1978 for some discussion of this). When calculating the absorption in a cloud one should take into account that a fraction of the radiation is scattered (albedo $\neq 0$ ). A Monte Carlo calculation of the effect of scattering on cloud penetration has been made by Sandell (1978). We shall very roughly approximate it by making a very conservative estimate. At $8 \mu \mathrm{m}^{-1}$ the albedo of the core-mantle grains is $\approx \frac{1}{2}$ and the albedo of the very small particles is $\simeq 0$. Since the contribution to the total extinction by the small particles at $\lambda^{-1}=8 \mu \mathrm{m}^{-1}$ is about $\frac{1}{3}$, the albedo of all the particles taken together is estimated to be $\alpha \simeq \frac{2}{3} \times \frac{1}{2}+\frac{1}{3} \times 0=\frac{1}{3}$. Using this we get $A_{\mathrm{UV}}^{\mathrm{abs}} / A(V)=2$. Note that our value of $\alpha$ is substantially less than the albedo of $\sim 0.6$ which has been observed (Witt and Lillie, 1973). We let the attenuation in the cloud be given by this (apparently overestimated) grain absorption (see Table I)

$$
A(V)=0.54 \times 10^{-21} N_{\mathrm{H}}
$$

so that

$$
A_{\mathrm{UV}}^{\mathrm{abs}} \simeq 2 A(V)=1.1 \times 10^{-21} N_{\mathrm{H}},
$$

where $N_{\mathrm{H}}$ is the column density of hydrogen in all forms $\left(N_{\mathrm{H}}=N_{\mathrm{HI}}+2 N_{\mathrm{H}_{2}}\right)$.

Consider a uniform spherical cloud of radius $a$. At a distance $r$ from the center the ultraviolet absorption may be written as

$$
A_{\mathrm{UV}}^{\mathrm{abs}}(r)=1.1 \times 10^{-21}(a-r) n_{\mathrm{H}},
$$

where $n_{\mathrm{H}}$ is the volume density of hydrogen. The criterion for effective photolysis is then

$$
\tau_{\mathrm{ph}}(r)=\tau_{\mathrm{ph}}^{\mathrm{IM}} \exp \left\{A_{\mathrm{UV}}^{\mathrm{abs}}(r) / 1.086\right\} \leqslant \tau_{\mathrm{ff}},
$$

where $\tau_{\mathrm{ph}}^{\mathrm{IM}}$ is the photolysis time in the general interstellar medium, $\tau_{\mathrm{ff}}$ is the free-fall time, and where we have used the optical depth in terms of the astronomical extinction: $A / 1.086 \equiv \Delta m / 1.086=$ optical depth.

Let $\tau_{\mathrm{ph}}^{\mathrm{IM}}=1000 \mathrm{yr}$ and

$$
\tau_{\mathrm{ff}}=\frac{4 \times 10^{7}}{n_{\mathrm{H}}^{1 / 2}} \mathrm{yr} .
$$


TABLE I

Ultraviolet penetration in clouds

\begin{tabular}{|c|c|c|c|}
\hline \multirow{2}{*}{$\begin{array}{l}\text { Optical depth } \\
\text { at cloud center } \\
\text { at } \lambda^{-1}=8 \mu \mathrm{m}^{-1} \\
\tau(8)\end{array}$} & \multirow{2}{*}{$\begin{array}{l}\text { Absorption depth } \\
\text { at cloud center } \\
\text { at } \lambda^{-1}=8 \mu \mathrm{m}^{-1} \\
\begin{aligned} \tau_{(8)}^{\text {abs }}=2 \tau(V) \\
\quad=\frac{2}{3} \tau(8)\end{aligned} \\
\text { (see Equation 2) }\end{array}$} & \multicolumn{2}{|c|}{ Penetration at cloud center } \\
\hline & & $\begin{array}{l}\text { this paper } \\
\exp \left\{-\tau_{(8)}^{\text {abs }}\right\}\end{array}$ & $\begin{array}{l}\text { Sandell (1978) } \\
\alpha=0.6 \\
g=0.75\end{array}$ \\
\hline 1 & 0.67 & $5.12 \times 10^{-1}$ & $6.7 \times 10^{-1}$ \\
\hline 2 & 1.33 & $2.64 \times 10^{-1}$ & $4.36 \times 10^{-1}$ \\
\hline 5 & 3.33 & $3.57 \times 10^{-2}$ & $8.92 \times 10^{-2}$ \\
\hline 10 & 6.67 & $1.27 \times 10^{-3}$ & $5.18 \times 10^{-3}$ \\
\hline 20 & 13.33 & $1.62 \times 10^{-6}$ & $5.86 \times 10^{-5}$ \\
\hline
\end{tabular}

Then Equation (4) becomes

$$
\tau_{\mathrm{ph}}(r)=10^{3} \exp \left\{3\left(\frac{n_{\mathrm{H}}}{10^{3}}\right)\left(\frac{a-r}{p c}\right)\right\} \leqslant 1.3 \times 10^{6}\left(\frac{n_{\mathrm{H}}}{10^{3}}\right)^{-1 / 2}
$$

Solving this equation for $a-r=1 \mathrm{pc}$ we get (conservatively) the maximum cloud density to be $n_{\mathrm{H}} \simeq 2 \times 10^{3} \mathrm{~cm}^{-3}$. For $a-r=0.1 \mathrm{pc}$, the solution is $n_{\mathrm{H}} \simeq 2 \times 10^{4} \mathrm{~cm}^{-3}$. Grain photolysis remains substantial throughout a $1 \mathrm{pc}$ radius cloud whose total visual extinction is about $5 \mathrm{mag}$ or to a penetration of $0.1 \mathrm{pc}(30 \%$ of the volume of a 1 pc cloud) within a cloud having a visual extinction of 50 mag. Since we have underestimated the ultraviolet penetration in dense clouds by a substantial factor relative to the more exact calculations of Sandell (see Table I), our density estimates are too small. In view of this, and the fact that dense clouds are normally prevented from collapsing in times as short as their free fall times, it is fair to state that grain photolysis is important during almost the entire cycle-time of grains even while they are in very dense clouds.

A previous comparison of the photoprocessing rate with the grain accretion rate

TABLE II

Comparison between laboratory and interstellar conditions

\begin{tabular}{|c|c|c|}
\hline & $\mathrm{Lab}$ & ISM \\
\hline Mantle $\left\{\begin{array}{l}\text { 'Initial' composition } \\
\text { Thickness } \\
\text { Temperature }\end{array}\right.$ & $\begin{array}{l}\text { (simple molecules) } \\
\mathrm{CO}, \mathrm{H}_{2} \mathrm{O}, \mathrm{NH}_{3}, \mathrm{CH}_{4} \cdots \\
\geqslant 0.1 \mu \mathrm{m} \\
\gtrsim 10 \mathrm{~K}\end{array}$ & $\begin{array}{l}\text { All interstellar condensible } \\
\quad \text { species } \\
\sim 0.15 \mu \mathrm{m} \\
\gtrsim 10 \mathrm{~K}\end{array}$ \\
\hline Gas $\left\{\begin{array}{l}\text { Pressure of condensibles } \\
\text { Number of condensible species }\end{array}\right.$ & $\begin{array}{l}8 \times 10^{-8} \mathrm{mbar} \\
2.4 \times 10^{9} \mathrm{~cm}^{-3}\end{array}$ & $\begin{array}{l}3 \times 10^{-17} \mathrm{mbar} \\
1 \mathrm{~cm}^{-3}\left(n_{11}=10^{3} \mathrm{~cm}^{-3}\right)\end{array}$ \\
\hline Ultraviolet $\left\{\begin{array}{c}\Phi_{\mathrm{UV}} \equiv \text { Flux }(E>6 \mathrm{eV} \\
\lambda<2000 \AA) \\
\text { Equivalent time scales }\end{array}\right.$ & $\begin{array}{l}10^{15} \mathrm{~cm}^{-2} \mathrm{~s}^{-1} \\
1 \mathrm{hr}\end{array}$ & $\begin{array}{l}10^{8} \mathrm{~cm}^{-2} \mathrm{~s}^{-1} \\
10^{3} \mathrm{yr}\end{array}$ \\
\hline
\end{tabular}


(Greenberg, 1976) should also be modified to account for the proper ultraviolet penetration. If this is done, we note (see Equation 13, ibid.) that in the center of a $1 \mathrm{pc}$ cloud with $n_{\mathrm{H}}=10^{4} \mathrm{~cm}^{-3}$ we have substantial photoprocessing relative to accretion, which means that even as the grains grow they are still subject to significant chemical modification, storing of radicals and subsequent ejection. It should also be noted that we have been conservative in considering only dense clouds without sources. If the cloud has a direct source of ultraviolet radiation, such as provided by a hot star, or an indirect source of ultraviolet radiation, such as provided by $\mathrm{T}$ Tauri winds (Norman and Silk, 1979), the conditions for grain photolysis are even more clearly seen to be universally prevalent.

Of course all of the above time scales are impossibly long for laboratory measurements so that we must scale up the ultraviolet flux enough to provide substantial effects in times of the order of hours or days. In Table II we show schematically the basic relative conditions in the laboratory and in space.

The thickness and the temperature of the mantle material can be made the same in the laboratory as in space. The initial chemical composition, given similar relative abundances of $\mathrm{O}, \mathrm{C}$ and $\mathrm{N}$, should be unimportant if the total radiation time is long enough to completely modify the mixture.

The photon flux in the laboratory is relatively high but not high enough to produce spurious results. For example, the possibility that a photon will strike a particular molecule while it is in an excited state produced by a previous photon is negligible. The time between two photons interacting with the same molecule is, for a $t>0.1 \mu \mathrm{m}$ thick sample,

$$
\tau \simeq \frac{t}{\Phi_{\mathrm{UV}} d^{3}} \simeq 1000 \mathrm{~s}
$$

where the molecule diameter $d$ is taken as $\sim 2 \times 10^{-8} \mathrm{~cm}$. Molecular relaxation times are generally many orders of magnitude less than this.

Although the gas density in the laboratory is much larger than in space, nevertheless its photochemical effects are negligible compared to those in the solid because the total number of line-of-sight gas molecules is, in the laboratory configuration, less than $10^{-9}$ that of the molecules in a $0.1 \mu \mathrm{m}$ solid layer.

We have thus demonstrated not only that photochemical processing of interstellar grain mantles plays a dominant role in grain evolution but also that the laboratory results may be used directly to provide a quantitative description of these effects and their consequences to molecule formation.

\section{General Experimental Procedure and Instrumental Details}

The general proccdure consists of three steps, each step analyzed by a variety of diagnostic methods:

(1) The grain mantle is simulated by a gas mixture deposited (accreted) on a cold $(10 \mathrm{~K})$ substrate (e.g., aluminum mirror or sapphire window). 


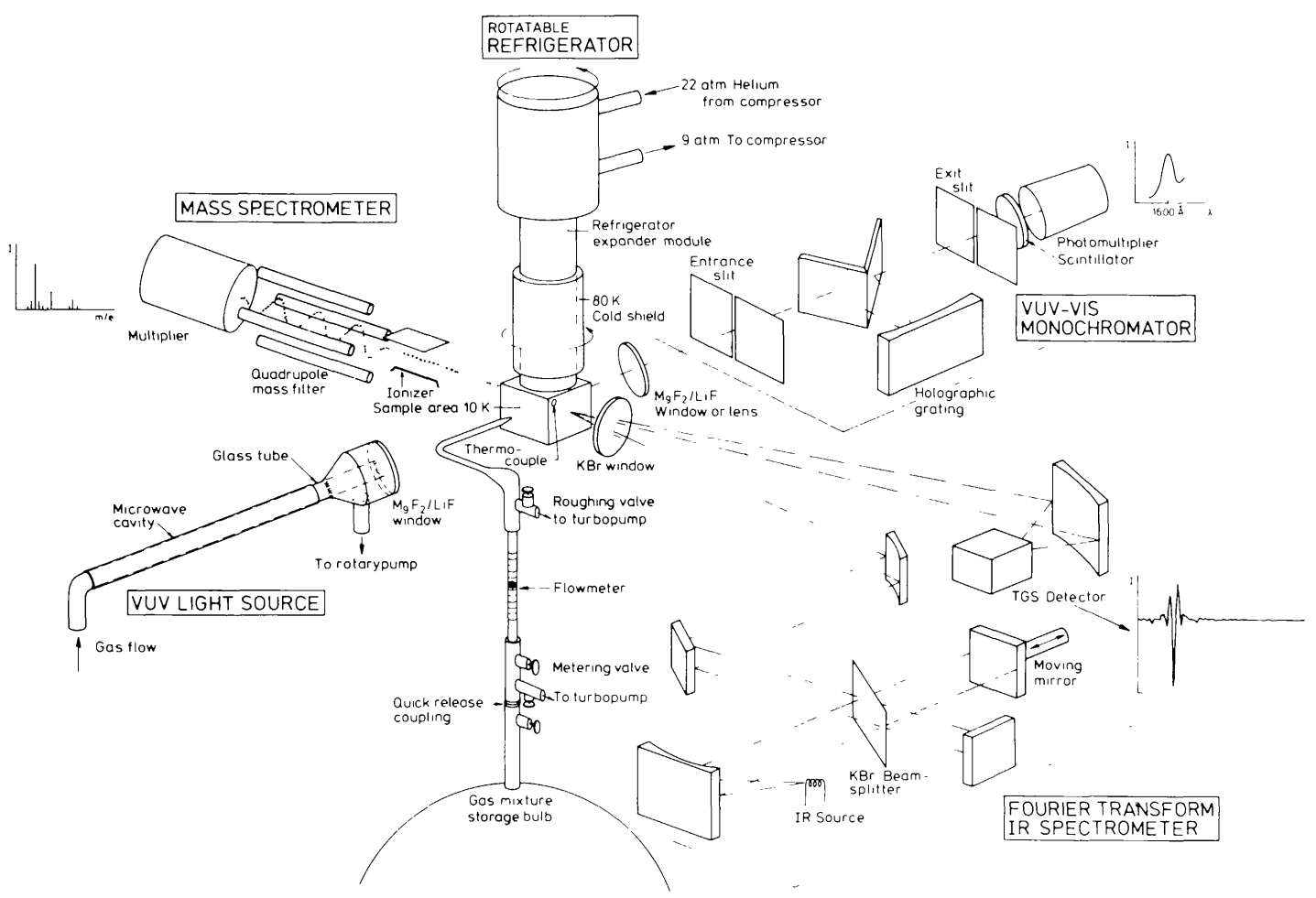

Fig. 1 Schematic diagram showing the orientation of the equipment used in sample preparation and detection.

(2) The frozen gas mixture is subjected to vacuum ultraviolet radiation produced by, e.g. a microwave powered hydrogen flow lamp. Photons penetrate the sample and cause photochemical reactions in the bulk of the material such as the formation of radicals.

(3) The cold sample is allowed to warm up. Stored reactive species formed during photlysis react and give rise to luminescence. As the temperature of the substrate rises various components of the mixture evaporate.

The chemical processing of the grain mantle analog is followed with infrared (IR) absorbance spectrometry (steps 1, 2, 3), mass spectrometry $(1,3)$, ultraviolet-visible (UV-vis) spectrometry $(1,2,3)$ and the observation of UV and visible phosphorence (2) and chemiluminescence (3).

The reader is referred to Figure 1 throughout the following description of the instrumental details.

\section{(a) Low Temperature Sample Substrate}

The cold $(10 \mathrm{~K})$ substrate for the grain mantle analog is either a window (e.g., sapphire, $20 \mathrm{~mm}$ diam.) mounted in an electrolytic copper holder, or a polished aluminum block $\left(2 \times 2 \times 2 \mathrm{~cm}^{3}\right)$ which acts as a mirror. The four sides of the block can be used for four sequential experiments without cleaning the mirror. This eliminates a time con- 
suming warm up after every experiment and occasional breaking of the vacuum for removal of the reaction products. The advantages of such a four-sided reflector are described in more detail elsewhere (Nibler, 1976).

\section{(b) Refrigeration}

The sample substrate is kept at a temperature of $10 \mathrm{~K}$ (or higher) by a closed cycle helium refrigerator (Air Products Displex CSW-202A + DMX 1AE - cooling capacity $0.5 \mathrm{~W}$ at $12 \mathrm{~K}$, or Cryomech GB04-1 W at $10 \mathrm{~K}$ ). The normal operating temperature of $10 \mathrm{~K}$ is reached after cooling for $90 \mathrm{~min}$ and can be maintained indefinitely. The temperature is measurable and continuously adjustable up to about $330 \mathrm{~K}$ by reducing the helium pressure and/or by electric heating. The temperature is measured with a $\mathrm{Au}-\mathrm{Fe} 4 \%$ Chromel thermocouple mounted at the window holder or mirror. These are connected to the cold tip with an indium seal for improved thermal conductivity. The cold tip is rotatable inside the vacuum shroud, thus allowing the

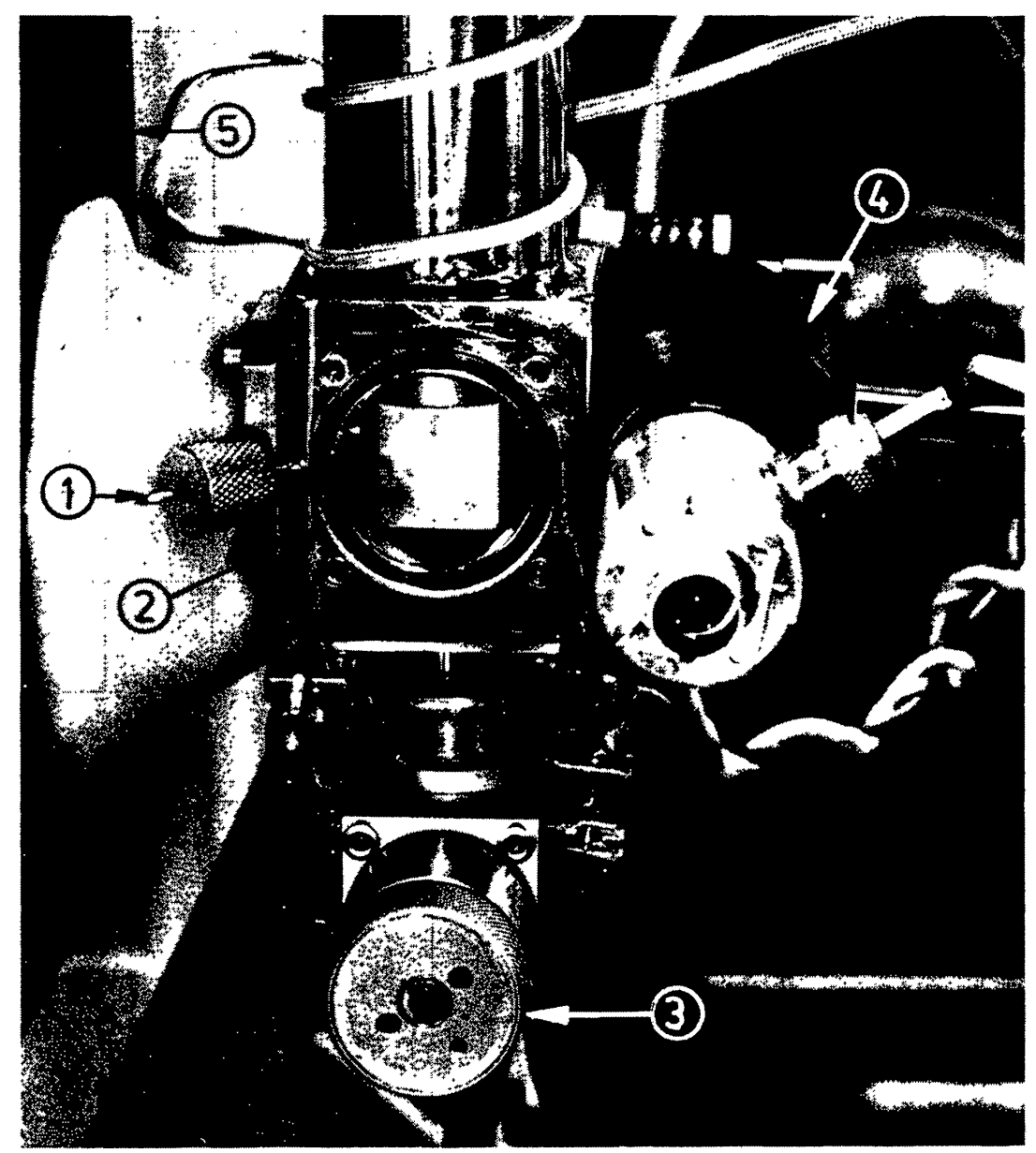

Fig. 2. Close-up of sample chamber showing: (1) gas deposition port (not used), (2) rotatable aluminum mirror (note the Newton Rings, indicating the presence of a sample), (3) valve leading to mass spectrometer, (4) photomultiplier tube for total luminescence measurements, (5) infrared spectrometer. 
mirror or window to face towards the sample deposition line, the vacuum light source, the IR optics and the UV-vis optics. The cold tip and its vacuum shroud can be isolated and detached from the vacuum system once the temperature is low enough for sufficient cryopumping.

\section{(c) High Vacuum System}

The stainless steel vacuum system is partly built up from Pneurop quick release couplings with Viton O-rings, evacuated by an oil free turbomolecular pump (LeyboldHeraeus, L-H, Turbovac, pumping speed $200 \mathrm{ls}^{-1}$ ) backed by a rotary pump $\left(10 \mathrm{~m}^{3} \mathrm{~h}^{-1}\right)$. A sorption trap filled with aluminum oxide pellets and/or a liquid nitrogen cold trap minimize oil entry from the rotary pump into the high vacuum system. The pressure in the high vacuum system is measured using a $\mathrm{L}-\mathrm{H}$ Combivac IT 20 power supply which controls a hot cathode ionization gauge in combinations with a pyrani gauge. The system provides a working, oil free vacuum of $2-8 \times 10^{-8}$ mbar.

\section{(d) Gas Handling Manifold}

The gas mixtures, of which the ice mantle analogs will be composed, are prepared in a greaseless glass vacuum line using standard gas handling techniques. Briefly it consists of a glass manifold using greaseless O-ring stopcocks (9 and $16 \mathrm{~mm} \mathrm{diam}$.) with glass O-ring joints (Louwers Hapert) or metal O-ring compressing connectors (Cajon). Gas pressures can be measured with two stainless steel diaphragm type meters (range 0-1000 and 0-100 mbar), a U-shaped manometer filled with silicon diffusion pump oil and a pyrani-ionization gauge combination as described in the high vacuum section. Vacuum is obtained by a liquid nitrogen trapped oil diffusion pump (L-H Leybodiff 170, $140 \mathrm{ls}^{-1}$, filled with Dow Corning 704 pumping fluid) backed by a rotary pump. Under normal conditions this system is operated at $4 \times 10^{-5}$ mbar (the gas mixtures prepared usually have pressures $>100 \mathrm{mbar}$ ).

\section{(e) Sample Preparation}

The grain mantle analog is prepared by bleeding a gas mixture into the high vacuum system through a capillary tube $(1 \mathrm{~mm}$ I.D.) directed to a face of the substrate at a distance of $1 \mathrm{~cm}$. The gas immediately freezes onto the cold substrate. No pressure rise is observed, indicating that almost all the gas is actually condensed onto the cold tip. The gas flow from a storage bulb to the vacuum system is controlled with a flow meter (Fischer \& Porter 10 A 1017 A) and regulated with a needle valve.

All results presented here are for mixtures of $\mathrm{NH}_{3}, \mathrm{H}_{2} \mathrm{O}$ and $\mathrm{CO}$, which are deposited on the cold substrate at a rate of about $0.5 \mathrm{~m} \mathrm{Mol} \mathrm{h}^{-1}$. Gases are at room temperature. The ammonia (Matheson, anhydrous, 99.96\%) and methane (Matheson, Ultra High Purity, 99.995\%) are used without further purification. Water is purified by triple distillation, followed by several freeze-pump-thaw cycles carried out under

vacuum. The carbon monoxide (Matheson, Matheson purity, 99.997\%) is filtered 
through molecular sieves (Linde, $13 \mathrm{X}$ ) and activated charcoal (Merck, $2.5 \mathrm{~mm}$ pellets) prior to use.

\section{(f) Light Sources for Photolysis}

The vacuum UV light sources for the photochemistry experiments are microwave (in some cases RF) powered discharge lamps similar in design and performance to those previously described by Harteck et al. (1932, 1964), Warneck (1962), Okabe (1963) and Thompson et al. (1965). In the core of the flow lamp a gas or gas mixture is led through a pyrex tube in an air-cooled McCaroll microwave cavity (Fehsenfeld et al., 1964, 1965; manufactured by Opthos Instr. Co.). The $2450 \mathrm{MHz}$ microwave power is generated by a magnetron (Philips 7090. max. output $200 \mathrm{~W}$, run at $1.6 \mathrm{kV}$, $50 \mathrm{~mA})$ powered by a current stabilized high voltage supply. The lamp window $\left(\mathrm{MgF}_{2}\right.$ for use down to $1150 \AA$, LiF to $1070 \AA$ ) is mounted in a quick release O-ring fitting. The gas supply line is $\frac{1}{+}$ in copper tubing. The gas flow is maintained by a liquid nitrogen trapped rotary pump. The pressure, which is measured with a pyrani gauge, and the mixing ratio (for gas mixtures) are regulated by diaphragm valves combined with needle valves (Hoke 1335 G4B and LH Variable Leak 283 30). The source most frequently used to date is the undiluted hydrogen (Matheson Extra Dry, 99.95\%) flow lamp, which has a molecular emission system around $1600 \AA$, shown in Figure 3.

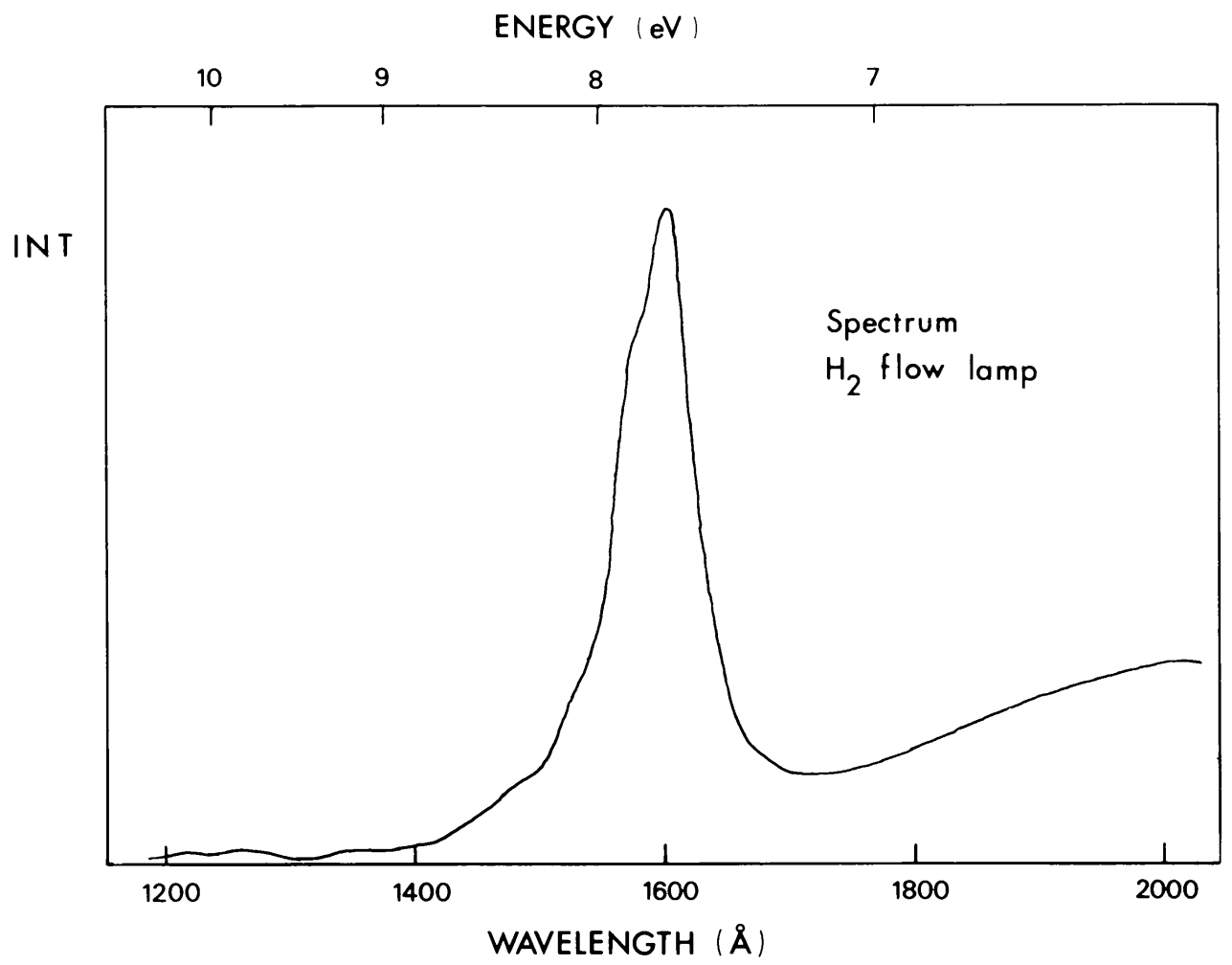

Fig. 3. Spectrum ( $20 \AA$ resolution) obtained from the hydrogen flow lamp when equipped with $\mathrm{MgF}_{2}$ window and operated at 0.2 mbar. Note that the dominant emission is centered at $1600 \AA$. 
Maximum output at $1600 \AA$ is obtained at a $\mathrm{H}_{2}$ pressure of $1-3 \times 10^{-1}$ mbar. The absolute vacuum UV flux is $1.5 \times 10^{15}$ photons $\mathrm{s}^{-1}$ as measured by $\mathrm{CO}_{2}$ actinometry (Thompson et al., 1965; Slanger et al., 1974).

\section{(g) Analytical Methods}

Infrared absorption spectra $\left(4000-500 \mathrm{~cm}^{-1}\right.$ or $\left.2.5-20 \mu \mathrm{m}\right)$ are measured with a Michelson interferometer (Digilab FTS $15 \mathrm{~B} / \mathrm{D}$, maximum resolution $0.25 \mathrm{~cm}^{-1}$, with Nova 2 minicomputer operating on a Digilab software package based on the Data General RDOS system). The infrared beam is reflected by the aluminum mirror on which the sample is deposited, so that the sample is passed through twice. Experiment has shown that reflection at the sample-vacuum surface is negligible for most purposes but not when the absorbance is strong. Infrared absorbance spectra give good qualitative and quantitative information on the changes occurring in the sample during photoprocessing because most molecular species have several vibrational bands of well defined wavelength and relative intensity in this spectral region.

Ultraviolet and visible absorbance spectra (2000-10 $000 \AA)$ of the sample (deposited on a sapphire window) are measured in transmission using a combination of tungsten light source, monochromator (Spex Industries, model 1870, $0.5 \mathrm{~m}, \mathrm{f} 6.9,16 \AA \mathrm{mm}^{-1}$, grating blazed at $5000 \AA$ ) and photomultiplier tube (Hamamatsu R $212 \mathrm{UH}$, S5 photocathode, range 2000-6000 $\AA$ ) or silicon photodiode (Hamamatsu, 51087-01, 5000-10 000 $\AA$ ). For luminescence and phosphorence a Jarrell Ash (model 82-410, $0.25 \mathrm{~m}, \mathrm{f} 3.6,33 \AA \mathrm{mm}^{-1}$, grating blazed at $6000 \AA$ ) monochromator is used.

Vacuum UV spectra (1000-2000 $\AA$ ) of the light sources are obtained with a vacuum monochromator (Jobin Yvon H 20 UVL, holographic grating 1000-5000 $\AA, 40$ $\AA \mathrm{mm}^{-1}, 1200$ grooves $\mathrm{mm}^{-1}$ ), mounted in a self built evacuable housing pumped by two liquid nitrogen adsorption pumps (L-H ASP 20 using zeolite filling) or by the turbomolecular pump. In this case the detector is a sodium salicylate coated photomultiplier tube (Hamamatsu R $212 \mathrm{UH}$ ). The coating process and many other instrumental details of VUV spectroscopy are described by Samson (1967).

Mass spectra of the volatile components of the photoprocessed sample are measured by a quadrupole mass spectrometer (Riber QMM 17, resolution $0.5 \mathrm{amu}$, range 1$300 \mathrm{amu}$, with electron multiplier). The data are manipulated via a microprocessor system (Motorola M6800) and recorded on casette tape. For larger masses we have access to mass spectrometers at the Institute of the Foundation for Fundamental Research on Matter (F.O.M., Amsterdam) and the Chemistry Department at Leiden.

\section{Systems and Results}

We are carrying out a wide variety of experiments on systems ranging from very simple single component samples to more complicated multi-component mixtures. In this report we limit ourselves to results of experiments which bear on some key phases

in the life of a grain mantle. Particular emphasis is given to experiments which provide 
an understanding of the role grain mantles play in interstellar molecule formation and destruction.

\section{(a) Infrared Studies}

The infrared spectrum from $4000-400 \mathrm{~cm}^{-1}(2.5-25 \mu \mathrm{m})$ of 0.73 millimoles of a $\mathrm{CO}: \mathrm{NH}_{3}: \mathrm{H}_{2} \mathrm{O}: \mathrm{CO}_{2}$ (50:1:1:0.09) mixture deposited over a period of 2 hours is shown with assignments in Figure 4. The region from 1000 to $1900 \mathrm{~cm}^{-1}$ of the same sample before and after 2 and 6 hours of in situ vacuum ultraviolet irradiation is presented in Figure $5 \mathrm{a}, \mathrm{b}$ and $\mathrm{c}$ respectively. Examination of this figure shows the appearance, stabilization and growth of the reactive species: HCO (formyl radical), $\mathrm{NH}_{2}$ (amino radical) and $\mathrm{HOCO}$ as well as the appearance and growth of the stable species $\mathrm{H}_{2} \mathrm{CO}$ (formaldehyde), $\mathrm{HCOOH}$ (formic acid) and $\mathrm{NH}_{2} \mathrm{HCO}$ (formamide). $\mathrm{CO}_{2}\left(2350\right.$ and $\left.667 \mathrm{~cm}^{-1}\right)$ and $\mathrm{HNCO}\left(2288 \mathrm{~cm}^{-1}\right)$, both not shown, are also found to grow. In addition new unassigned features are evident at 1045, 1290 and $1305 \mathrm{~cm}^{-1}$. All the identified new species formed are listed in Table III along with their interstellar counterparts. The rate of growth of $\mathrm{HCO}, \mathrm{H}_{2} \mathrm{CO}$ and $\mathrm{NH}_{2}$ slows down considerably after the first 2 hours while the gradual growth of a more complex molecule such as $\mathrm{NH}_{2} \mathrm{HCO}$ apparently continues at roughly the same rate. These different growth rates

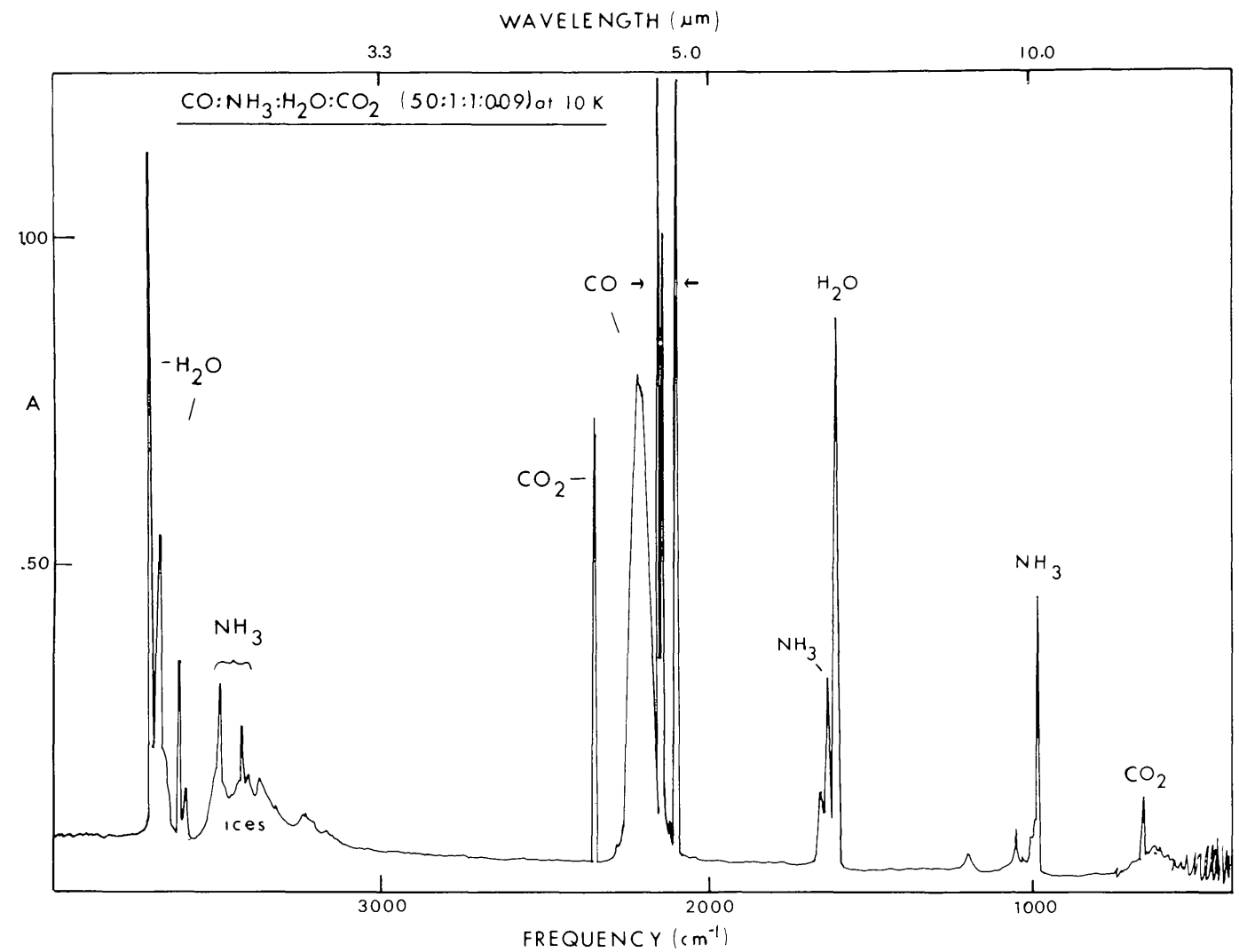

Fig. 4. The infrared spectrum of a mixture of $\mathrm{CO}: \mathrm{NH}_{3}: \mathrm{H}_{2} \mathrm{O}: \mathrm{CO}_{2}(50: 1: 1: 0.09)$ at $10 \mathrm{~K}$ prior to photolysis. References $-\mathrm{H}_{2} \mathrm{O}$ : van Thiel et al., 1957; $\mathrm{NH}_{3}$ : Pimentel et al., 1962; CO: Dubost, 1976. 


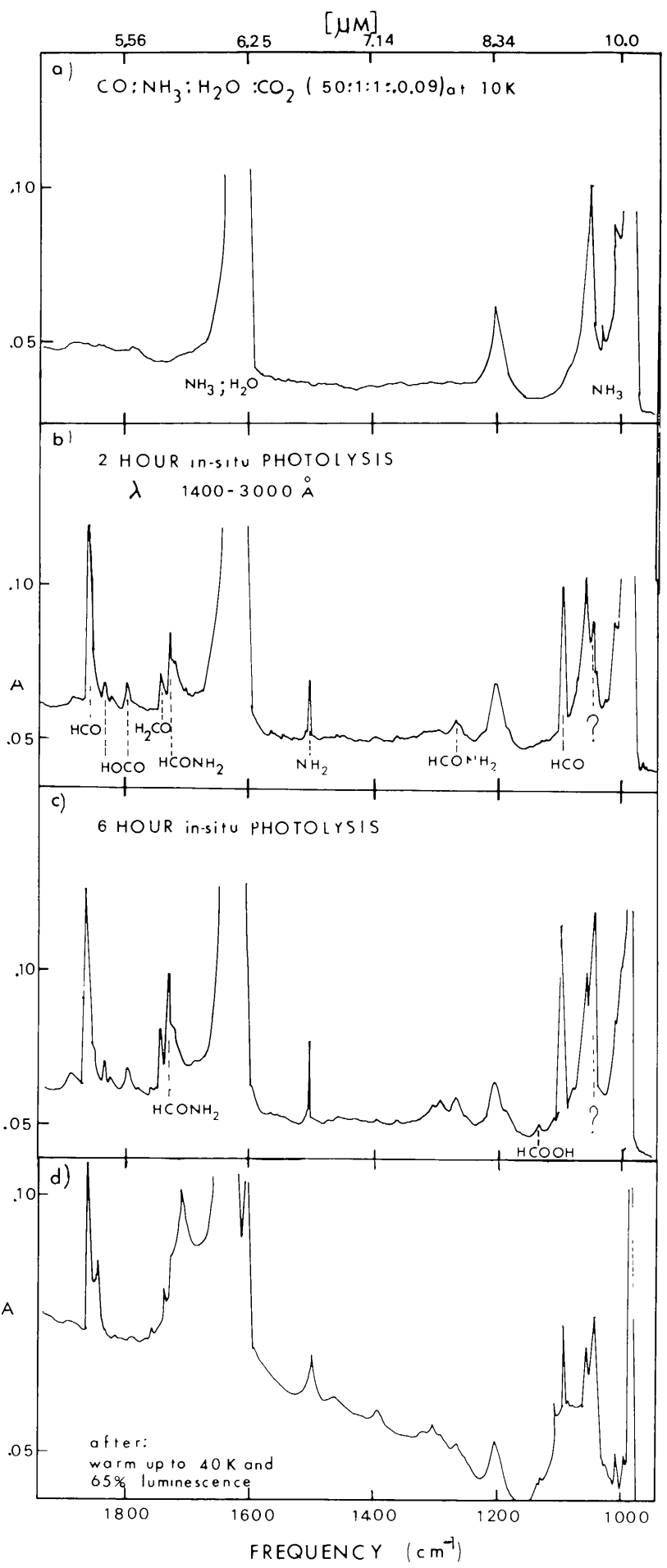

Fig. 5. Expansion of the Fig. 4 infrared spectrum showing the effects of photolysis (b, c) and warm-up (d) on the $\mathrm{CO}: \mathrm{NH}_{3}: \mathrm{H}_{2} \mathrm{O}: \mathrm{CO}_{2}$ mixture. References - $\mathrm{HCO}\left(2488,1860\right.$ and $\left.1091 \mathrm{~cm}^{-1}\right)$, Ewing et al., 1960; Milligan and Jacox, 1964); $\mathrm{NH}_{2}\left(3213\right.$ and $1499 \mathrm{~cm}^{-1}$, Milligan and Jacox, 1965): $\operatorname{HOCO}\left(1833,1797\right.$ and $1261 \mathrm{~cm}^{-1}$, Milligan and Jacox, 1971); $\mathrm{H}_{2} \mathrm{CO}(2861,2794,1736$ and $1497 \mathrm{~m}^{-1}$, Thomas and Guillory, 1973); $\mathrm{HCOOH} \mathrm{(1758} \mathrm{and} 1129 \mathrm{~cm}^{-1}$, Milligan and Jacox, 1971); $\mathrm{NH}_{2} \mathrm{HCO}$ (1726 and $1267 \mathrm{~cm}^{-1}$, Milligan and Jacox, 1965). 
TABLE III

Comparison of new species formed (solid) upon irradiation of the starting mixture $\mathrm{CO}: \mathrm{NH}_{3}: \mathrm{H}_{2} \mathrm{O}: \mathrm{CO}_{2}$ with those detected in the interstellar medium (gas)

\begin{tabular}{ll}
\hline Grain mantle analog & Interstellar medium \\
\hline $\mathrm{HCO}$ & $\mathrm{HCO}$ \\
$\mathrm{CO}_{2}$ & \\
$\mathrm{NH}_{2}$ & \\
$\mathrm{HNCO}$ & $\mathrm{HNCO}$ \\
$\mathrm{H}_{2} \mathrm{CO}$ & $\mathrm{H}_{2} \mathrm{CO}$ \\
$\mathrm{HOCO}$ & \\
$\mathrm{HCONH}$ & \\
$\mathrm{HCOOH}$ & $\mathrm{HCONH}$ \\
\hline
\end{tabular}

a Gammon, 1978.

are shown in Figure 6 where the absorbance of spectral features (free from overlap with other absorptions) for a few of the species present are plotted versus photolysis time. The relative absorbance values do not represent relative abundances of the different species since the absorption coefficients are not the same for the various modes plotted. For example, while the strength of the $\mathrm{CO}_{2}$ absorption at $2350 \mathrm{~cm}^{-1}$ is comparable to the $\mathrm{H}_{2} \mathrm{O}$ absorption at $1600 \mathrm{~cm}^{-1}$ in Figure 4, the relative amount of $\mathrm{CO}_{2}$ to $\mathrm{H}_{2} \mathrm{O}$ is only about 0.09 because the band strength for $\nu_{3}$ of $\mathrm{CO}_{2}$ is about $2500 \mathrm{~cm}^{-2} \mathrm{~atm}^{-1}$, nine times greater than that for $\nu_{2}$ (about $280 \mathrm{~cm}^{-2} \mathrm{~atm}^{-1}$ ) in $\mathrm{H}_{2} \mathrm{O}$ (Pugh and Rao, 1976).

The spectral changes which take place in the sample after it was slowly warmed up to $40 \mathrm{~K}$ and recooled can be seen by comparing Figure $5 \mathrm{c}$ with $5 \mathrm{~d}$. This comparison shows a reduction in absorption by the radicals $\mathrm{NH}_{2}$ and $\mathrm{HCO}$ with simultaneous growth of formaldehyde $\left(\mathrm{H}_{2} \mathrm{CO}\right)$ and formamide $\left(\mathrm{NH}_{2} \mathrm{HCO}\right)$. The disappearance of the radicals and growth of the more stable molecules is expected since mild warming allows diffusion to take place thus permitting the unstable species to react and produce stable product molecules.

Another important application of the infrared measurements is the comparison of laboratory produced spectra with those obtained from observations. The infrared absorption band around $3200 \mathrm{~cm}^{-1}(3.1 \mu \mathrm{m})$ is of special interest because many interstellar infrared sources show a prominent absorption feature at about this wavelength usually attributed to $\mathrm{H}_{2} \mathrm{O}$ ice (Gillett et al., 1975; Merrill et al., 1976; Cohen, 1976; Capps et al., 1978; Harris et al., 1978). Up to now no laboratory measurements of low temperature ices have reproduced the observed spectrum. While some laboratory spectra have maximum absorption at the right frequency, they do not account for the band width at half height nor the wing at the low frequency side of the observed feature without invoking additional assumptions such as unusually large grain sizes 


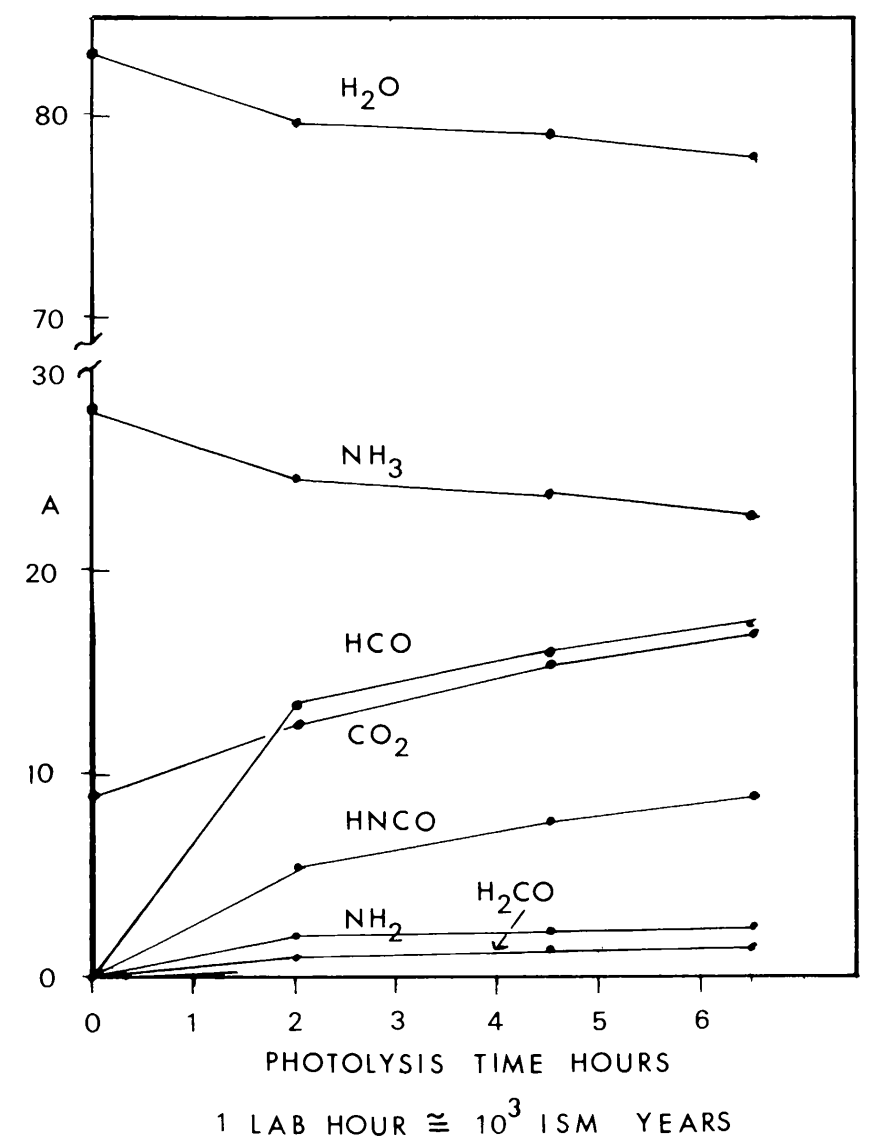

Fig. 6. Relative peak absorbances for a number of species present in the $\mathrm{CO}: \mathrm{NH}_{3}: \mathrm{H}_{2} \mathrm{O}: \mathrm{CO}_{2}$ (50:1:1:0.09) mixture as a function of in-situ photolysis time. Note that $1 \mathrm{~h}$ of photolysis in the laboratory is roughly equivalent to $10^{3} \mathrm{yr}$ in the interstellar medium, see Section 2 .

(Hunter and Donn, 1971; Merrill et al., 1976; Mukai et al., 1978; Capps et al., 1978). In Figure 7 we show the spectrum of a mixture of $\mathrm{CO}: \mathrm{H}_{2} \mathrm{O}(10: 1)$ after 2 hours of photolysis at $10 \mathrm{~K}$, warm up to $42 \mathrm{~K}$ and subsequent recooling. An example of the interstellar band as well as an absorption spectrum of amorphous $\mathrm{H}_{2} \mathrm{O}$ ice $\left(I_{a}\right)$ are shown for comparison. The $\mathrm{CO} / \mathrm{H}_{2} \mathrm{O}$ spectrum, arising naturally from the application of our ideas on grain evolution reflects all characteristics of the interstellar feature measured at $3200 \mathrm{~cm}^{-1}$. We are currently studying this absorption band in more mixtures and incorporating the spectrum into a detailed analysis of the infrared extinction and polarization by interstellar grains in dense clouds.

\section{(b) Visible Studies}

Absorption. The absorption spectrum between 3500 and $10000 \AA$ for a number of mixtures has been measured. In all cases the region between 4000 and $7000 \AA$ shows the most structure and is presented for the case $\mathrm{CO}: \mathrm{H}_{2} \mathrm{O}(40: 1)$ deposited at a rate of 0.5 millimoles $\mathrm{h}^{-1}$ for 22 hours in Figure 8 . These absorptions appear only in photolyzed samples. The strong, broad absorptions grow weaker upon warm up and are 


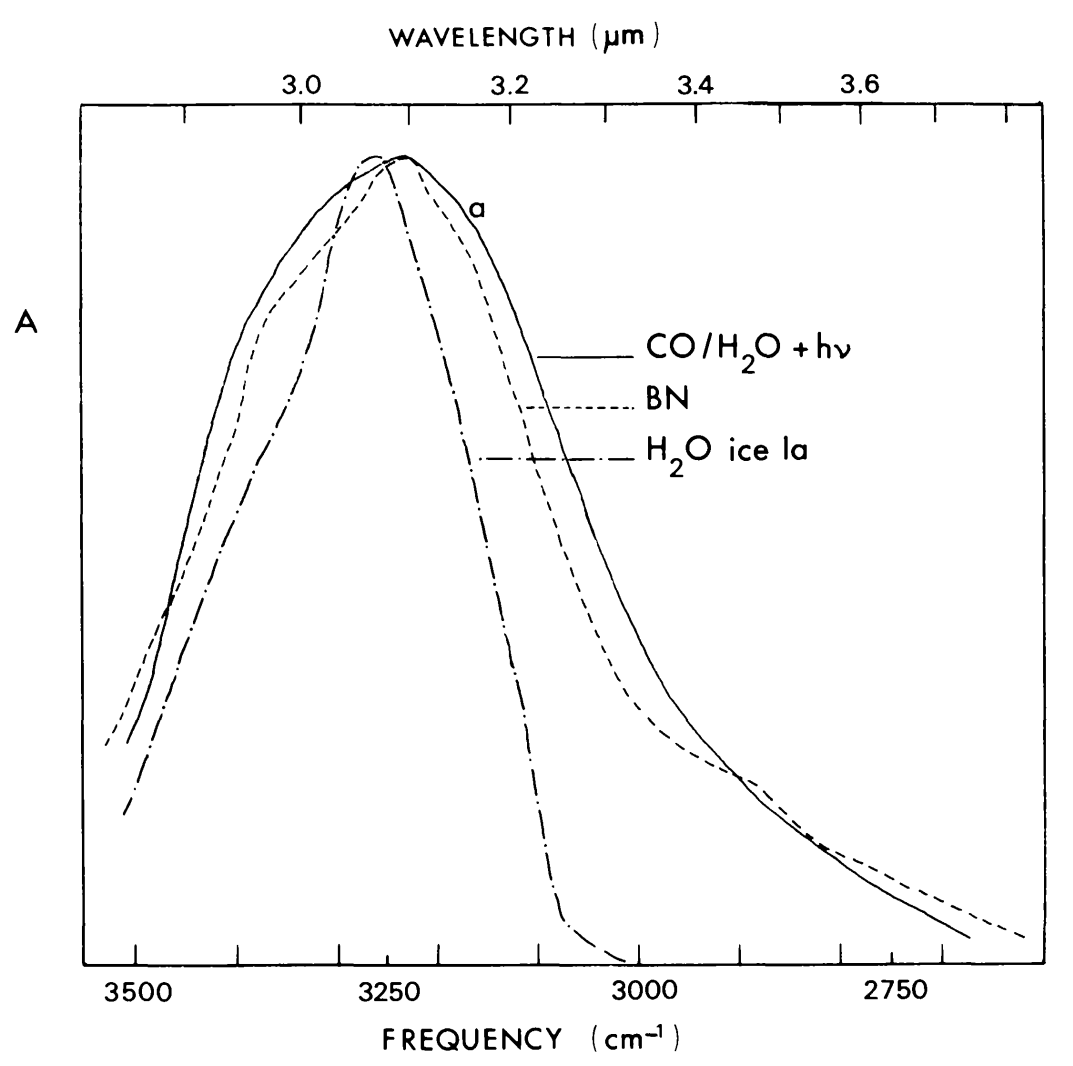

Fig. 7. The infrared spectrum in the region of $3200 \mathrm{~cm}^{-1}(3.1 \mu \mathrm{m})$ for $-\mathrm{CO}: \mathrm{H}_{2} \mathrm{O}(10: 1)$ measured at $10 \mathrm{~K}$ after $2 \mathrm{~h}$ in-situ photolysis at $10 \mathrm{~K}$ and subsequent warm-up to $42 \mathrm{~K}$; ----- the

BN object (Gillett et al., 1975); -...- amorphous $\mathrm{H}_{2} \mathrm{O}$ ice at $80 \mathrm{~K}$ ( $I_{a}$; Buontempo, 1972).

barely detectable once the sample has reached $35 \mathrm{~K}$. A portion of the spectrum had been reported earlier and was assigned to the HCO radical (Ewing et al., 1960). The disappearance of the features upon warm-up, the agreement with the features previously assigned to $\mathrm{HiO}$, the consistent energy differences between adjacent lines and the constant shift with respect to the gas phase transitions (Herzberg and Ramsey, 1955; Johns et al., 1963) allow a confident assignment to the radical HCO. We observe this spectrum in a large number of experiments even with starting mixtures as complex as $\mathrm{CO}: \mathrm{H}_{2} \mathrm{O}: \mathrm{NH}_{3}: \mathrm{CH}_{4}(10: 1: 1: 0.5)$. In all cases the absorptions are very broad (80-100 $\AA$ at half height).

Emission. As mentioned previously a number of irradiated samples luminesce upon warm-up. The spectra emitted from two different samples, $\mathrm{NH}_{3}: \mathrm{CO}(1: 100)$ and $\mathrm{H}_{2} \mathrm{O}: \mathrm{CO}(1: 100)$ measured at different temperatures are presented in Figure 9. The spectrum of the luminescence is roughly constant throughout, undergoing a gradual change in detail between the onset temperature of $10 \mathrm{~K}$ and $45 \mathrm{~K}$ where the emission stops.

While the spectrum observed from a variety of different mixtures is approximately the same, slight differences can be seen. This phenomena is now the object of intensive investigation in our laboratory. 
W. HAGEN ET AL.

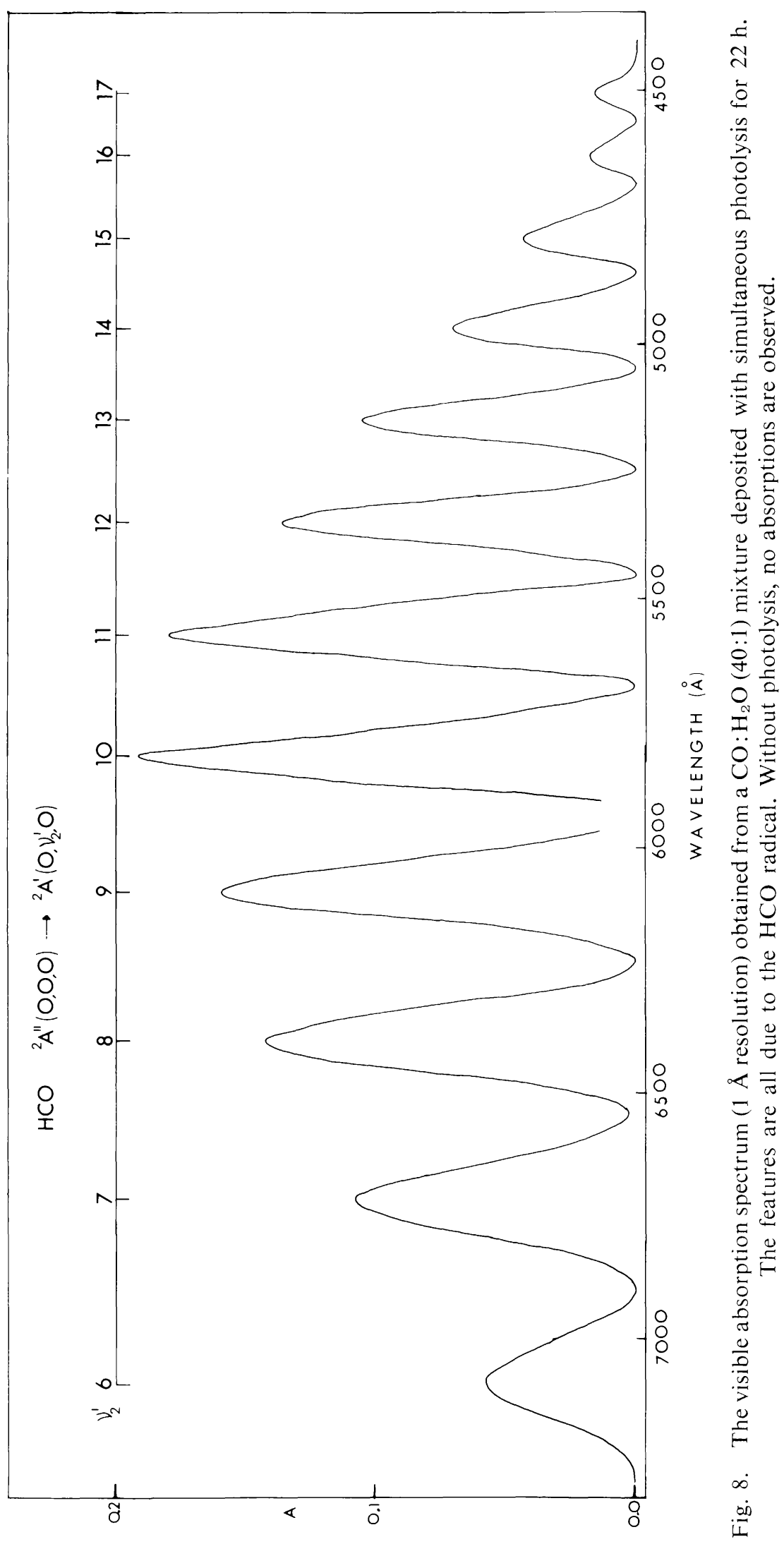




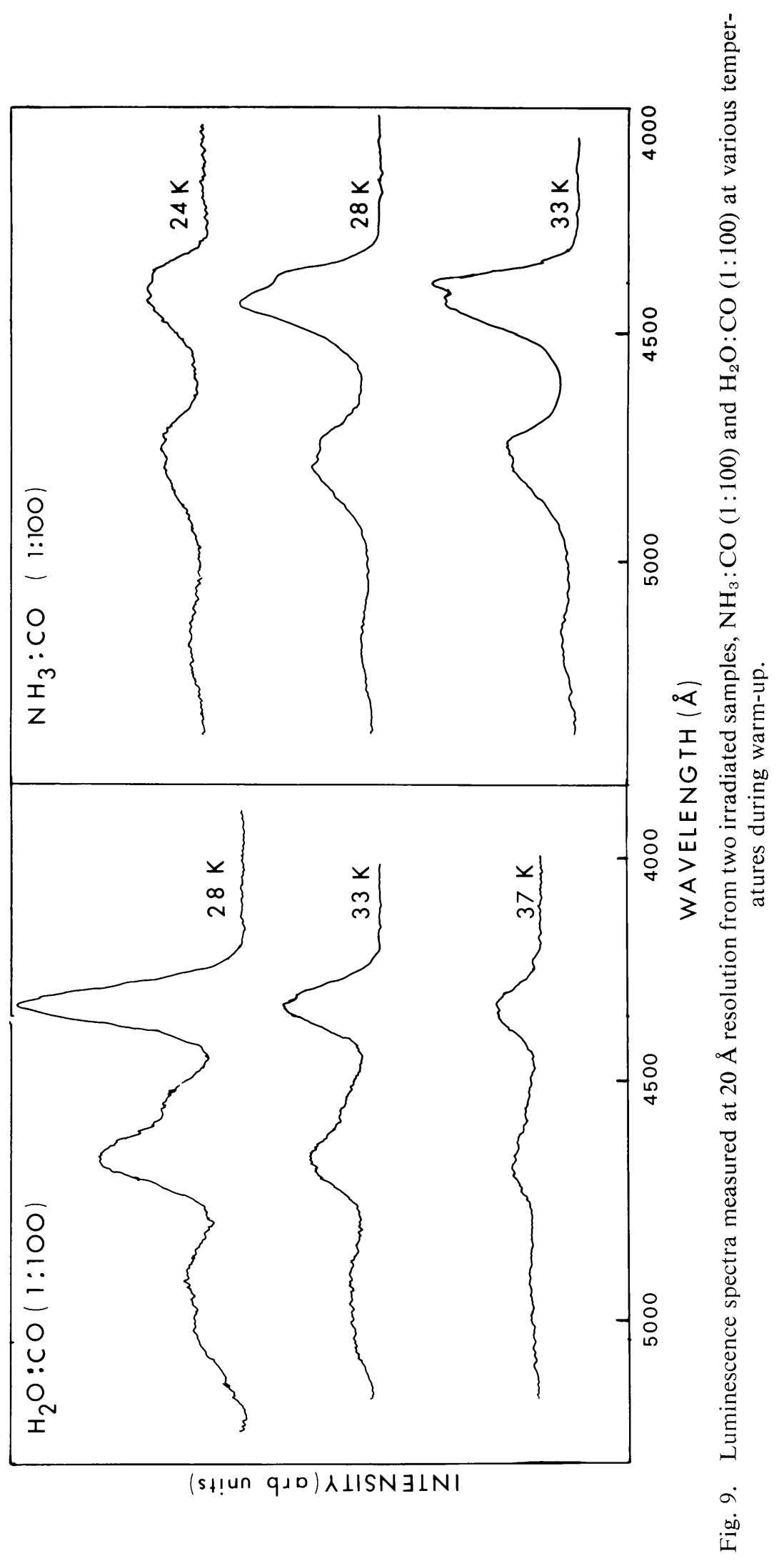




\section{(c) Vapor Pressure Studies}

Irradiated samples have been found to possess slightly enhanced vapor pressure curves in the very low temperature regime. Pressure versus temperature curves for an irradiated (solid line) and non-irradiated sample (dashed line) are shown in Figure 10. Examination of this figure shows that the pressure enhancement is a function of photolysis time with the greatest effect observed for the longest photolysis time. At temperatures higher than about $30 \mathrm{~K}$ the vapor pressure of the irradiated sample is indistinguishable from that of the unirradiated sample.

\section{(d) High Molecular Mass Studies}

Upon completion of a number of experiments starting with mixtures of $\mathrm{NH}_{3} / \mathrm{CO}$, $200 \mu \mathrm{g}$ of a viscous, yellow, water soluble fluid was recovered from the substrate which

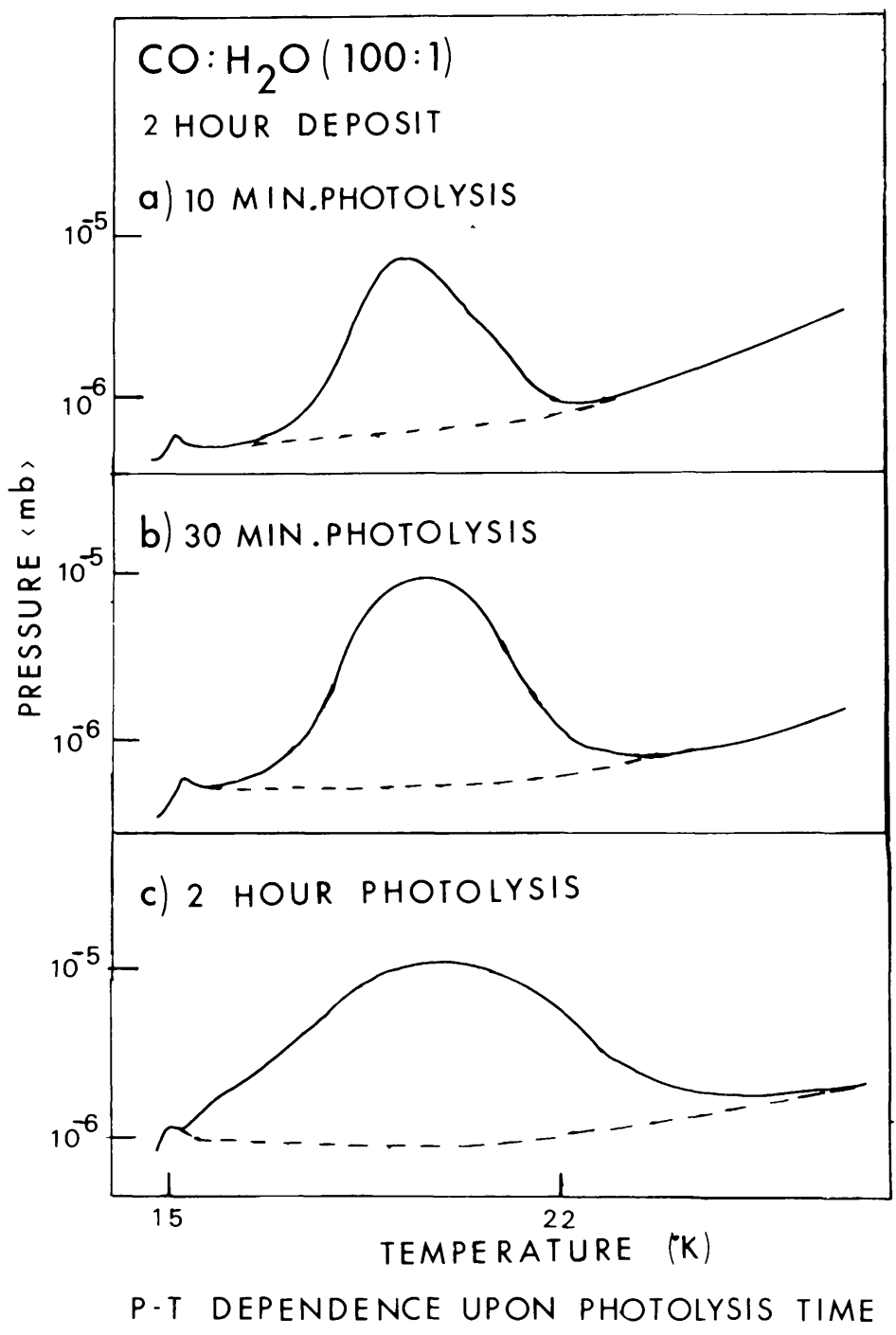

Fig. 10. Pressure as a function of temperature in the low temperature regime for a $\mathrm{CO}: \mathrm{H}_{2} \mathrm{O}$ (1:100) mixture showing the effect of in-situ photolysis time. The dashed line shows the curve obtained if an unirradiated sample is allowed to warm up. Above about $30 \mathrm{~K}$ the two curves are the same. 
represents a yield between $0.02 \%$ and $0.2 \%$. The mass spectrum of this substance has been obtained for us using laser and field desorption mass spectrometric techniques, both of which yielded a mass of 514 amu. In addition, the infrared spectrum of the yellow substance shows features characteristic of amino and carboxylic acid subgroups, but is inconsistent with hydrocarbon or aromatic compounds. The liquid evaporates between 400 and $500 \mathrm{~K}$. Under somewhat different conditions we have produced a residual yellow solid for which we have little data. Upon heating this in a mass spectrometer $\mathrm{CO}_{2}$ is readily liberated, characteristic of carboxylic acids. Furthermore the major portion of the material did not evaporate but pyrolyzed at $600 \mathrm{~K}$ !

In order to rule out the possibility of contamination the following observations are important. These experiments have been repeated more than six times in our two different cryostats and the residues obtained appear the same. When other mixtures are used, residues differing in appearance are found. For example $\mathrm{CH}_{4} / \mathrm{CO}$ mixtures result in a white residue which remains when the substrate has been heated to $350 \mathrm{~K}$. Again, more detailed investigations are in progress and will be reported separately.

\section{Discussion}

There must be three distinct steps if the grain mantles are an integral part of the interstellar molecule formation and destruction scheme. First, atoms and molecules must accrete and form a mantle; second, this mantle must undergo photochemical processing via absorption of interstellar radiation which results directly in the formation of a more varied array of molecules; and third, there must be a mechanism for the release of some of the new molecules back into the gas phase where the majority have been detected. We shall now discuss these points in the light of our experimental results.

There is no doubt as to the occurrence of the first step. The second step, that of the generation of different types of astrophysically significant molecules (Table III) by photochemical reactions taking place within grain mantles, is demonstrated by the result of the infrared and visible absorption measurements. In spite of the fact that radicals such as $\mathrm{HCO}$ and $\mathrm{NH}_{2}$ are very reactive, they are found to be stored in complex mixtures where they must be in contact with potential reaction partners. In addition the residue remaining after a number of experiments shows that photoprocessing also provides a path for the formation of extremely large and very complex molecules. If we scale the laboratory rate of production of these molecules to the interstellar case (see Table II), we find that, in $7 \times 10^{6} \mathrm{yr}$, between $2 \%$ and $20 \%$ of the grain mantle materials have been converted into a rather refractory complex organic substance. The total mass of this substance is comparable with that required - in the form of the bare interstellar particles - to provide the far ultraviolet interstellar extinction (see for example Greenberg, 1978).

To complete the picture, the ejection mechanism of some of the newly formed molecules along with some of the original mantle constituents is demonstrable within 
the framework of the generally very mild grain warming events occurring in the interstellar medium. The enhanced vapor pressure curves of irradiated samples support this. The radiation induced increase in the vapor pressure between the temperatures of 10 and $25 \mathrm{~K}$ shows that only a slight increase in temperature results in an increase in the amount of material ejected over that found for non-irradiated samples. This implies that there is a release of internally stored energy which warms the sample locally and causes the positive pressure deviation shown in Figure 10. The source of this energy must be the exothermic recombination reactions of the various radicals trapped in the irradiated sample. The emission of luminescence, the disappearance of absorptions known to be due to radicals, and the pressure enhancement upon mild warming all support this explanation.

The evolution of a grain and its role as a sink and source of molecules is pictured as a continuing process within dense as well as tenuous clouds. The greater astronomical interest lies in the dark clouds because, there, both the processes of acretion and molecule ejection occur at a faster rate even though the photoprocessing rate is somewhat reduced relative to that prevailing in a 'standard' cloud. An individual grain is considered as one of a statisticat ensemble of similar grains. The accretion process is a slow but steady one, while the ejection process occurs sporadically but vigorously. Subsequent to a gradual period of accretion and ultraviolet photoprocessing the grain is subjected to some sort of triggering event. Such a triggering source may, for example, be provided by grain-grain collisions caused by the turbulence in molecular clouds. Collisions with velocities as low as $0.1 \mathrm{~km} \mathrm{~s}^{-1}$ (or even less) will raise the temperature of the grain by some 30 degrees thus leading to diffusion of radicals in the mantle and the generation of heat which evaporates some part of the mantle. This individual grain cools very quickly, again becoming a sink to the molecules which hit it, and resumes the accretion-photolysis cycle. Thus the three steps of accretion, photolysis and evaporation are envisioned as taking place sequentially in an individual grain, but simultaneously when averaged over all the grains. Perhaps one or two of the three steps dominates depending on the cloud parameters such as density, temperature, proximity to an $\mathrm{H}$ il region, internal energy sources, turbulence and so on.

An initial attempt has been made to understand the steady state distribution of grain mantle sizes (Hong and Greenberg, 1978) based on a statistical treatment of the accretion-evaporation balance (Greenberg, 1979b). From this theory we find a clue as to why we must find molecules in clouds where they are expected to accrete on the grains faster than they can be generated by gas phase and surface reactions. A way of parameterizing the ejection mechanism was defined so that we may begin to look for the relevant laboratory measurements and make calculations of the molecule production by grain mantles. A very important consequence of this is that it is not justified to ignore grain mantle accretion to arrive at steady (or quasi-steady) state results (for example, Mitchell et al., 1978), but rather it is essential to include grain accretion as one of the important intermediate steps in the molecule formation process. 


\section{Conclusions}

We have shown from theoretical arguments and laboratory analog experiments that the photoprocessing of the interstellar grain mantles provides an important path to the understanding of the production of polyatomic molecules in the interstellar medium. Three of the basic physical processes in grain mantles which have been demonstrated and which we are continuing to investigate are:

(1) Creation and storage of radicals in low temperature complex mixtures by ultraviolet radiation.

(2) Reactions among radicals and molecules in the solid.

(3) Release of stored chemical energy leading to evaporation of grain mantles by internal heating.

The laboratory results show that the grain mantle molecules undergo substantial evolution by sequential photolysis, warm-up, cool down, photolysis etc.

Grains in dense clouds are shown to be not merely passive bodies accreting existing molecules and atoms but rather are dynamic centers which both create and 'destroy' (capture) molecules, thus giving the basis for a steady - or quasi-steady - state distribution of molecular densities and grain mantle sizes. The relatively slow processes of molecule and atom accretion and ultraviolet photolysis are counterbalanced 'in the mean', by discrete energy releasing events which produce molecules in the gas phase. Of course, the released molecules can further interact with each other, with the photons and with the grains.

Significantly, by simulating grain evolution we have been able in a very natural way to reproduce quite accurately the observed shape and position of the interstellar $3.1 \mu \mathrm{m}$ band without invoking such exotic qualities as very large gain size or strange chemical composition.

The measured rate of production of substantial quantities of a relatively refractory molecular complex provide the stimulus for several very exciting speculations, two of which are being investigated further:

(1) Very small particles made up of refractory complex organic molecules may make a significant contribution to the far ultraviolet interstellar extinction.

(2) Radicals produced in the small complex molecular particles may have absorptions corresponding to some of the unidentified diffuse interstellar bands. This is inferred from the way radicals absorb in simpler ices (Allamandola et al., 1979).

A third speculation is that the abundance as well as the complexity of the molecules evolved from the photoprocessing of grain mantles give the possibility of supplying enough pre-biogeneic material to be considered along with other sources as one of the bases for the origin of life.

Finally we should mention the fact that, according to theories of the cold aggregation of comets from dense prestellar clouds or in the protostellar nebula, the material of the comet nucleus closely represents the material of the interstellar grains. Thus the 
chemical and physical composition of comet dust and gas would provide the most directly accessible information on the interstellar dust (Greenberg, 1979c). The exciting prospect is that the proposed joint NASA-ESA comet mission (including the Halley probe) contains experiments which could produce results relating to this model.

\section{Acknowledgements}

This work could not have been done without the excellent contributions to the design and construction of the equipment by E. de Kuijper and L. van As. We wish to acknowledge very significant collaboration with Fred Baas and Cyp van de Bult and many stimulating discussions with C. A. Norman and M. van Hemert. We also appreciate experimental help from W. Bijleveld, W. Mulder, R. Windhorst and L. van Ijzendoorn. Special thanks are due to Dr P. Kistemaker of the F.O.M. Institute for his strong and essential support in our mass spectroscopic work and Prof. J. van der Waals, J. Schmidt and A. van Strien for generously allowing us the use of the two visible-UV monochrometers with which all of our absorption and luminescence work has been done. One of us (W.H.) thanks Prof. A. Yencha for many helpful discussions and hospitality during a four month visit to his laboratory at S.U.N.Y. Albany, as well as the Netherlands Organization for the Advancement of Pure Research (Z.W.O.) for their financial aid (Grant no. 78 111).

\section{References}

Allamandola, L. J., Greenberg, J. M. and Norman, C. A.: 1979, Astron. Astrophys. (in press). Bash, F.: 1979, Astrophys. J. (in press).

Bass, A. M. and Broida, H. P. (eds.): 1960, Formation and Trapping of Free Radicals, Academic Press, New York.

Buontempo, U.: 1972, Phys. Letters 42A, 17.

Calvert, J. G. and Pitts, J. M. Jr: 1966, Photochemistry. John Wiley and Sons,

Capps, R. W., Gillett, F. C. and Knacke, R. F.: 1978, Astrophys. J. 226, 863.

Carey, F.: 1970, Knickerbocker New's, Associated Press, Albany, New York, Dec. $31,1$.

Cohen, M.: 1976, Astrophys. J. 203, 169.

Danielson, E. E., Woolf, N. J. and Gaustad, J. E.: 1965, Astrophys. J. 141, 116.

Donn, B.: 1960 in A. M. Bass and H. P. Broida (eds.), Formation and Trapping of Free Radicals, Chapter 11, Academic Press, New York, p. 347.

Dubost, H.: 1976, Chem. Phys. 12, 139.

Ewing, G. E., Thompson, W. E. and Pimentel, G. C.: 1960, J. Chem. Phys. 32, 927.

Fehsenfeld, F. C., Evenson, K. M. and Broida, H. P.: 1964, National Bureau of Standards Report 8701.

Fehsenfeld, F. C., Evenson, K. M. and Broida, H. P.: 1965, Rev. Scient. Instru. 36, 294.

Field, G. B.: 1978, in T. Gehrels (ed.), Protostars and Planets, Univ. of Arizona Press, Tucson.

Gammon, R. H.: 1978, Chem. Engng. News 56, No. 41, 21.

Gillett, F. C., Jones, T. W., Merrill, K. M. and Stein, W. A.: 1975, Astron. Astrophys. 45, 77.

Greenberg, J. M.: 1963, Ann. Rev. Astron. Astrophys. 15, 267.

Greenberg, J. M.: 1971, Astron. Astrophys. 12, 240.

Greenberg, J. M.: 1973, in M. A. Gordon and L. E. Snyder (eds.), Molecules in the Galactic Environment, John Wiley and Sons, p. 93. 
Greenberg, J. M.: 1976, Astrophys. Space Sci. 39, 9.

Greenberg, J. M.: 1978, in A. J. M. McDonnell (ed.), Cosmic Dust, Chapter 4, Wiley, London, p. 187.

Greenberg, J. M.: 1979a, The Moon and Planets 20, 15.

Greenberg, J. M.: 1979b, in B. Westerlund (ed.), Stars and Star Systems, IAU 4th European Regional Meeting in Astronomy, Reidel, Dordrecht, p. 173.

Greenberg, J. M.: 1979c, in W. I. Axford, H. Fechtig, J. Rahe (eds.), ESA Workshop on Cometary Missions, Bamberg, p. 119.

Greenberg, J. M., Yencha, A. J., Corbett, J. W. and Frisch, H. L.: 1972, Mem. Roy. Soc. Sciences de Liège, 6e série, Tome III, 425.

Hallam, H. E.: 1973, Vibrational Spectroscopy of Trapped Species, John Wiley and Sons, New York.

Habing, H. J.: 1968, Bull. Astron. Inst. Ned. 19, 421.

Harris, D. H., Woolf, N. J. and Rieke, G. H.: 1978, Astrophys. J. 226, 829.

Harteck, P. and Oppenheimer, F.: 1932, Z. Phys. Chemie B16, 77.

Harteck, P., Reeves Jr, R. R. and Thompson, B. A.: 1964, Z. Naturforschg. 19a, 2.

Herzberg, G. and Ramsay, D. A. 1955, Proc. Roy. Soc. (Lond.) A233, 34.

Hong, S. S.: 1975, Unified model of interstellar grains, Ph.D. Thesis, State Univ. of New York at Albany.

Hong, S. S. and Greenberg, J. M.: 1978, Astron. Astrophys. 70, 695.

Hunter, C. E. and Donn, B.: 1971, Astrophys. J. 167, 71.

Jackson, J. L.: 1959a, J. Chem. Phys. 31, 154.

Jackson, J. L.: 1959b, J. Chem. Phys. 31, 722.

Johns, J. W. C., Priddle, S. H. and Ramsay, D. A.: 1963, Discussions Faraday Soc. 35, 90.

Lindblad, B.: 1935, Nature 135, 133.

Merrill, K. M., Russell, R. W. and Soifer, B. T.: 1976, Astrophys. J. 207, 763.

Meyer, B.: 1971, Low Temperature Spectroscopy, Elsevier Press, New York.

Miller, S.: 1953, Science 117, 528.

Milligan, D. E. and Jacox, M. E.: 1964, J. Chem. Phys. 41, 3032.

Milligan, D. E. and Jacox, M. E.: 1965, J. Chem. Phys. 43, 4487.

Milligan, D. E. and Jacox, M. E.: 1971, J. Chem. Phys. 54, 927.

Mitchell, G. F., Ginsburg, J. L. and Kuntz, P. J.: 1978, Astrophys. J. Suppl. 38, 39.

Mukai, T., Mukai, S., and Noguchi, K.: 1978, Astrophys. Space Sci. 53, 77.

Nibler, J. W.: 1976, Private communication; see also Lesiecki, M. L.: 1975, Spectroscopic studies of high temperature matrix isolated molecules, Ph.D. Dissertation, Oregon State University.

Norman, C. A. and Silk, J.: 1979, Astrophys. J. (in press).

Okabe, H.: 1963, J. Opt. Soc. Am. 54, 478.

Oort, J. H.: 1974, in J. R. Shakeshaft (ed.), Recent Studies of Bright Galaxies, IAU Symp. No. 58, Reidel, Dordrecht, p. 375.

Pimentel, G. C., Bulanin, M. O. and van Thiel, M.: 1962, J. Chem. Phys. 36, 500.

Pugh, L. A. and Rao, K. N.: 1976, in K. Rao (ed.), Molecular Spectroscopy, Modern Research, Academic Press, New York, Vol. II, p. 165.

Salpeter, E. E.: 1977, Ann. Rev. Astron. Astrophys. 15, 267.

Samson, J. A. R.: 1967, Techniques of Vacuum Ultraviolet Spectroscopy, Wiley, New York, p. 213. Sandell, G.: 1978, Astron. Astrophys. 69, 85.

Slanger, T. G., Sharpless, R. L., Black, G., Filseth, S. V.: 1974, J. Chem. Phys. 61, 5022.

Snyder, L. E., Buhl, D., Zuckerman, B. and Palmer, P.: 1969, Phys. Rev. Letters $22,679$.

Spitzer, L. Jr.: 1969, Diffuse Matter in Space, Wiley, New York.

ter Haar, D.: 1943, Bull. Ast. Inst. Ned. 10, No. 361, 1.

Thomas, Jr., S. G. and Guillory, W. A.: 1973, J. Phys. Chem. 77, 2469.

Thompson, B. A., Reeves, Jr, R. R. and Harteck, P.: 1965, J. Phys. Chem. 69, 3964.

van de Hulst, H. C.: 1943, Ned. Tijdschr. v. Natuurkunde 10, 251.

van de Hulst, H. C.: 1949, Recherche Astron. Observatoire Utrecht 11, part 2.

van Thiel, M., Becker, E. D. and Pimentel, G. C.: 1957, J. Chem. Phys. 27, 486. 
Warneck, P.: 1962, Appl. Optics 1, 721.

Watson, W. D.: 1976, Rev. Mod. Phys. 48, 513.

Witt, A. N. and Johnson, M. W.: 1973, Astrophys. J. 181, 363.

Witt, A. N. and Lillie, C. F.: 1973, Astron. Astrophys. 25, 371.

Zuckerman, B. and Evans, N. J. II: 1974, Astrophys. J. 192, L149.

Note added in proof: We have received a preprint from A. Leger, J. Klein, S. De Cheveigne, C. Guinet, D. Defourneau and M. Belin (Research Note, Astron. Astrophys., in press) in which it is demonstrated that the central position, as well as the width of the $3.1 \mu \mathrm{m}$ feature, can be satisfactorily reproduced with amorphous ice. However, we have shown that the observed low frequency wing cannot be explained using amorphous ice alone (see Section 4(a) of this paper). 